\title{
Issues, Problems and Possibilities of Life Skills Education for School Going Adolescents
}

\author{
Prof. C. G. Venkatesha Murthy ${ }^{1 *}$
}

Keywords: Issues, Problems, Possibilities, Life Skill, Education, School, Adolescents

The present paper has two parts. Part A covers issues and problems of Life skills Education. Part B covers possibilities of life skills education.

\section{Part A: Issues and Problems of Life skills Education for school going adolescents}

\section{BACKGROUND ISSUES:}

Education is a process of accelerating the rate of maturation among children and adolescence. Both the childhood and adolescence are crucial stages of development, which will have to be channelized healthily in order to create a rich human resource. World Health Organization (WHO) defines individuals in the age group of 10-19 as adolescents, in the age group of 10-24 as young people and in the age group of 15-24 as youth (WHO 2007).

As part of Adolescent Education Programme, NCERT has come up with training and resource materials, (NPEP, 2010), where it holds that "one of the major objectives of adolescence education is to develop life skills to empower young people to respond to the real life situations in positive and responsible ways. Life Skills are psycho-social abilities that enable individuals to translate knowledge, attitude and values regarding their concerns into well-informed and healthy behaviors. Empowered with skills; young people are able to take decisions based on a logical process of "what to do, why to do, how to do and when to do."

It is important that the children and adolescents need to be enabled to grow as healthy and capable individuals. Preparing them only for their cognitive domain concerns would make them slant. They should eventually grow socially smart and worldly wise. Developing them for their psycho social competence is an important agenda before education. WHO, (2004) while advocating the promotion of psycho-social competence holds a view that "psychosocial

\footnotetext{
${ }^{1}$ RIE, Mysore, India

*Responding Author

(C) 2016 I V Murthy; licensee IJIP. This is an Open Access Research distributed under the terms of the Creative Commons Attribution License (http://creativecommons.org/licenses/by/2.0), which permits unrestricted use, distribution, and reproduction in any Medium, provided the original work is properly cited.
} 
competence is a person's ability to deal effectively with the demands and challenges of everyday life. It is a person's ability to maintain a state of mental well-being and to demonstrate this in adaptive and positive behaviour while interacting with others, his/her culture and environment.

Psycho-social competence has an important role to play in the promotion of health in its broadest sense; in terms of physical, mental and social well-being. In particular, where health problems are related to behaviour, and where the behaviour is related to an inability to deal effectively with stresses and pressures in life, the enhancement of psycho-social competence could make an important contribution. This is especially important for health promotion at a time when behaviour is more and more implicated as the source of health problems.

The most direct interventions for the promotion of psychosocial competence are those which enhance the person's coping resources, and personal and social competencies. In school-based programmes for children and adolescents, this can be done by the teaching of life skills in a supportive learning environment.”

Keeping in view the above, it is necessary to understand life skills education programme as proposed by WHO. According to WHO (2004) "Life skills are abilities for adaptive and positive behaviour, that enable individuals to deal effectively with the demands and challenges of everyday life.

Described in this way, skills that can be said to be life skills are innumerable, and the nature and definition of life skills are likely to differ across cultures and settings. However, analysis of the life skills field suggests that there is a core set of skills that are at the heart of skills-based initiatives for the promotion of the health and well-being of children and adolescents. These are listed below:

- Decision making

- Problem solving

- Creative thinking

- Critical thinking

- Effective communication

- Interpersonal relationship skills

- Self-awareness

- Empathy

- Coping with emotions

- Coping with stress 
Issues, Problems and Possibilities of Life Skills Education for School Going Adolescents

Decision making helps us to deal constructively with decisions about our lives. This can have consequences for health if young people actively make decisions about their actions in relation to health by assessing the different options, and what effects different decisions may have.

Similarly, problem solving enables us to deal constructively with problems in our lives. Significant problems that are left unresolved can cause mental stress and give rise to accompanying physical strain.

Creative thinking contributes to both decision making and problem solving by enabling us to explore the available alternatives and various consequences of our actions or non-action. It helps us to look beyond our direct experience, and even if no problem is identified, or no decision is to be made, creative thinking can help us to respond adaptively and with flexibility to the situations of our daily lives.

Critical thinking is an ability to analyse information and experiences in an objective manner. Critical thinking can contribute to health by helping us to recognise and assess the factors that influence attitudes and behaviour, such as values, peer pressure, and the media.

Effective communication means that we are able to express ourselves, both verbally and nonverbally, in ways that are appropriate to our cultures and situations. This means being able to express opinions and desires, but also needs and fears. And it may mean being able to ask for advice and help in a time of need.

Interpersonal relationship skills help us to relate in positive ways with the people we interact with. This may mean being able to make and keep friendly relationships, which can be of great importance to our mental and social well-being. It may mean keeping good relations with family members, which are an important source of social support. It may also mean being able to end relationships constructively.

Self-awareness includes our recognition of ourselves, of our character, of our strengths and weaknesses, desires and dislikes. Developing self-awareness can help us to recognise when we are stressed or feel under pressure. It is also often a prerequisite for effective communication and interpersonal relations, as well as for developing empathy for others.

Empathy is the ability to imagine what life is like for another person, even in a situation that we may not be familiar with. Empathy can help us to understand and accept others who may be very different from ourselves, which can improve social interactions, for example, in situations of ethnic or cultural diversity. Empathy can also help to encourage nurturing behaviour towards people in need of care and assistance, or tolerance, as is the case with AIDS sufferers, or people 
Issues, Problems and Possibilities of Life Skills Education for School Going Adolescents

with mental disorders, who may be stigmatized and ostracized by the very people they depend upon for support.

Coping with emotions involves recognising emotions in ourselves and others, being aware of how emotions influence behaviour, and being able to respond to emotions appropriately. Intense emotions, like anger or sorrow can have negative effects on our health if we do not react appropriately.

Coping with stress is about recognising the sources of stress in our lives, recognising how this affects us, and acting in ways that help to control our levels of stress. This may mean that we take action to reduce the sources of stress, for example, by making changes to our physical environment or lifestyle. Or it may mean learning how to relax, so that tensions created by unavoidable stress do not give rise to health problems.

Inevitably, cultural and social factors will determine the exact nature of life skills. For example, eye contact may be encouraged in boys for effective communication, but not for girls in some societies, so gender issues will arise in identifying the nature of life skills for psychosocial competence. The exact content of life skills education must therefore be determined at the country level, or in a more local context. However, described in general terms, life skills are being taught in such a wide variety of countries that they appear to have relevance across cultures" (WHO, 1997).

WHO's initiative is indeed laudable and it has been able to inform and influence the world on the importance of developing life skills among students. It is indeed a life-long process to mature and handle issues of life confidently. It is very important to realise that school education plays a crucial role in life skills development as everything happens by design in any school. It exposes students to varied experiences in the formative years and it has lot of advantages and perhaps in this endeavour school has a vantage point. Further, as proposed by WHO, Life skills are generic skills. It means, they are neither grade specific nor stage specific. It means life skills can be taught to children of any age and at any stage. They also need to be developed among adolescents.

Life skills education programme has been found to be effective in different settings "including programmes for the prevention of substance abuse (Botvin, et. al. 1980, 1984; Pentz, 1983) and adolescent pregnancy (Zabin et al. 1986; Schinke, 1984), the promotionf intelligence (Gonzalez, 1990) and the prevention of bullying (Olweus, 1990). Educational programmes teaching these skills have also been developed for the prevention of AIDS (WHO/GPA, 1994; Scripture Union, undated), for peace education (Prutzman et al., 1988) and for the promotion of self-confidence and self-esteem (TACADE, 1990). Teaching life skills in this wide range of promotion and 
prevention programmes demonstrates the common value of life skills for health promotion, beyond their value within any specific programmes.”

\section{Life Skills as an Integral Part of Adolescence Education:}

According to NPEP (2010) "the revised framework of Adolescence Education incorporates life skills as one of the competencies, perhaps the most critical competency developed and inculcated through education. It is generally believed that a person who is educated is equipped with all the needed abilities including life skills. But in reality this does not happen. Knowing what needs to be done or knowing what needs to be changed does not mean that the learners automatically know how to bring about behaviour changes. It is the Life skills that, if properly developed, provide the know-how and the tools to actualize behaviour change.

Life Skills in this context need to be defined as psycho-social abilities that enable individuals to translate knowledge, attitude and values regarding all the concerned issues into action. These may not be confined to only those related to health, mental health, sexual development, HIV and AIDS and Drug abuse. Life skills development empowers learners to observe the process involving 'what to do, why to do, how to do and when to do'. It encompasses the ability to build sound, harmonious relationships with self, others and the environment, the ability to act responsibly and safely, the ability to survive under a variety of conditions, and the ability to solve problems."

Under approach framework, NCERT (NPEP 2010) holds that "it is important to note that life skills development does not mean development of skills afresh by a set of educational interventions at a particular point of time. Life skills development is an integral part of the allencompassing process of socialisation that continues throughout human life. School education is an integral part of this process. In fact, individuals apply the acquired life skills in different contexts differently. An individual may have acquired a life skill and she/he may also be equipped with the ability to apply that skill in a context that is fundamentally different from adolescent reproductive and sexual health. For example, an adolescent may be applying thinking skill or communication skill very effectively while interacting with her/his teacher or even peer group during a discussion on say, globalization or environmental pollution, but she/he may not have the ability to apply those skills on an issue related to sex and sexuality or negative peer pressure. Life skills development, therefore, may be more aptly defined as a process of acquiring the ability to apply concerned skills in the specific context and not the development of that skill afresh.

Since life skills are generic by nature, an educational intervention aimed at the development of ability to apply them may be effective only when it is focused on the specific context. The intervention may have to be designed and operationalized differently for different contexts, more particularly for a culturally sensitive context like adolescent reproductive and sexual health 
concerns. It needs interventions to focus on acquisition of authentic knowledge, development of positive attitude, and empowerment for avoidance of risky behavior.

The design of educational intervention has to take note of the content area and also specific life skills. Since most of the contents of adolescence education are very sensitive, interventions need to be well conceived for doing justice to the content. Contextually relevant and age appropriate contents should be focused on. It is important to exactly identify which life skills are to be focused and also the reasons for doing so.

In order to organize educational interventions for life skills development effectively, it is important to identify curricular as well as co-curricular activities that have the potential for developing skill application ability. Certain specific activity will be more appropriate than others in respect of a particular life skill. For example, role play can be very appropriate in respect of negotiation skills or interpersonal skills or skills related to empathy. One activity can be organized to attain different objectives. It is the process of organizing that activity that makes a fundamental difference by providing exact direction for attaining the desired objective. Group discussion may be able to attain knowledge, understanding and even attitude related objectives, but if it is to attain skill development related objectives, it has to be planned and conducted according to a particular process that sustains its focus throughout on skill development. Since life skills development primarily depends through the mode of experiential learning mode, the process of involving learners in the activity is very important.”

WHO (1997), proposes that life skills education as generic skills should include practice of skills in relation to major health and social problems. Life skills lessons should be combined with health information, and may also be combined with other approaches. As regards the methods, it proposed that life skills should build around own experiences and experiences around them. Life skills sessions should involve dynamic teaching and learning process. The methods could include brainstorming, role play, games and debates. It further proposes that life skills education programme for adolescents could cover concerns related to the prevention of drug abuse and teenage pregnancy, the promotion of mental well-being and cooperative learning. Ideally, life skills education should begin early before the influence of negative patterns of behaviour and interaction. The school is an appropriate place for the introduction of life skills education because of:

- the role of schools in the socialization of young people;

- access to children and adolescents on a large scale

- economic efficiencies (uses existing infrastructure);

- experienced teachers already in place;

- high credibility with parents and community members;

- possibilities for short and long term evaluation. 
In a school situation, it is important to secure long term plan, support and resources for life skills education. Life skills education needs to be provided from the beginning. Therefore, life skills education programme will require training packages/ teaching manuals. This investment is desirable and necessary. Since life skills programme are to be used for the promotion of health and wellbeing, it should cover all children and adolescents, rather than as an intervention aimed only at those already at risk or who already have problems. Adolescents seem to be most vulnerable to behaviour-related health problems.

The above issues relating to Life skills education has caught the attention of different countries of the world. It is also bearing fruits. In a vast country like India, there is a need to introduce life skills education programme by preparing the system sufficiently. However, there are certain problems in the introduction of life skills education in India.

\section{PROBLEMS INVOLVED IN THE INTRODUCTION OF LIFE SKILLS EDUCATION:}

There are problems involved in the introduction of life skills education and as a result, it has not taken roots in this India as much as it should have. There are variations in understanding and handling life skills by different people differently. Some of them are elaborated. These are the personal views of the author.

(a) Conceptual and procedural issues: It is not out of place to say that there are indeed conceptual and procedural issues associated with life skills education. Different people involved with life skills education programme view them differently. Even though there are only 10 life skills proposed by WHO, it has been stretched beyond also. WHO proposed that life skills are generic skills. It should mean that they are not age-specific or stage-specific or gender-specific. But, there are packages meant for specific classes! In terms of procedures too different people follow different procedures. There are attempts to develop more than one skill in one activity too! Thus there are conceptual and procedural issues associated with life skills education. There is a need to harmonize these issues.

(b) Stand alone v/s Integrated approach: Another very big issue is in treating life skills education programme as either stand alone or to develop in an integrated approach. There are two groups of thought. Developing life skills as stand-alone can have a structured format. While proposing life skills education as an integrated approach has its own basic issues. Since class room teachers are so heterogeneous in nature and teaching styles differ so much, expecting an integrated approach becomes very difficult to achieve. There cannot be any one method in which teachers can be enabled to integrate. Developing any training strategy for a wide variety of teachers teaching different subjects on integrated approach also becomes too difficult. Ideally, all teachers need to understand the significance of developing life skills and use it appropriately in their teaching learning activities. They would be able to do it once they understand all issues surrounding life skills education programme. One can make some exemplars also. BUT 
preparing training packages for teachers on how life skills can be developed in an integrated manner amount to restricting teachers to move from a natural style of transaction to an artificial style. This is the danger it may have. Therefore, training teachers at the first level to understand issues surrounding life skills education could be done using a stand-alone and dedicated training package. The integration issues should be left to the teachers themselves.

(c) Life skills education as an ancillary programme: Many managements view life skills education as an ancillary programme. Achieving highest marks or CGPA in board examination is real achievement for many. Any activity which can take away time from this goal of marks or grades become ancillary. The obsession with marks/grade oriented education system does not consider anything other than that as substantive. Thus, it becomes ancillary! This attitude must change. There is also a need to persuasively negotiate with all the managements about the objective of working towards all-round development of children as education. In this context, life skills education must find its legitimate place and dignity in the scheme of things.

(d) Lack of consensus of Life Skills Education Programme (LSEP): There is no consensus about Life Skills Education programme in the country. As a result different packages are getting generated. There is a need for consensus on many issues of life skills education programme. Professional organizations can do it. This is the need of the hour. There is a need for a national consultation on conceptualising and executing life skills education programs. Once, a national model is achieved, it needs to be disseminated widely so that more and more students can benefit. This should be a national priority. In fact this could be one of the recommendations of this conference.

(e) LSEP has taken off differently already: Since Life skills Education programme has already taken off differently by different persons/ organizations/NGOs. It may not be easy to bring in concensus, unless it is initiated at the national level. In the name of life skills education programmes many activities are taking place. There are variations at different levels. How I wish I was wrong.

(f) LSEP needs acceptable mandate and methodology at national level: It is necessary to have a national acceptable mandate of the life skills Education programme in the country. In the fitness of things, I have no hesitation in reiterating that, there is a need to have a national level consultation about bringing a training model at the nation level. There is also a need to suggest methodology which is doable by all teachers in the country without demanding any additional resources. The sooner it is done, the better it is for the country. Therefore, finding out solutions to the above problems need to be done urgently and it should be construed as an agenda for needed reforms in this area. 


\section{Part B: Possibilities of Life skills Education for school going adolescents}

Keeping in view the broad objectives of adolescent education programme, WHO's concerns about life skills education and the problems discussed above, there is a need to discuss the possibilities of life skills education programme logically and persuasively. Perhaps, the following dimensions would help us to synthesize the possibilities.

(a) Common philosophical premise: WHO has proposed that life skills are to be construed as generic skills. Therefore, they can neither be age/stage specific nor class/gender specific. Further, ALL children need to be trained for life skills. It is important to note that no skill can be developed per se. There is a need to study different components underlying each skill which need to be carefully delineated and activities are to be planned around them. Life skills education programme need to satisfy the above premise.

(b) Non negotiable principles: Some of the non negotiable principles could include the following.

(i) No skill can be developed per se. They need to be broken down into components. Each component needs to be operationally defined in the context of the skill. Development of each component has to be concern over a period of time.

(ii) For each component, a number of interventions need to be planned.

(iii) The proposed interventions must be easily doable by all teachers without any requirement of extra resources. Therefore, some of the common interventions could include Brain storming activities, Role plays and Group discussions.

(iv) A common basic training package needs to be developed with national level approval for wider use.

(v) The training package needs to be developed for key resource persons and teachers for the first level.

(vi) A training package and training in a certain way meant for integration of life skills into every day teaching learning would amount to robbing the teacher initiatives in developing life skills through subject teaching.

(vii) The training package must be a generic package which could be used for all children at all levels.

(c) Easily doable methodology: The present author believes that there are three levels of conceiving life skills training package. The first level training package must aim at exclusively introducing different life skills and their components. This should be meant for master trainers and teachers. After they understand and develop some clarity and conviction, at the second level, teachers can conduct exclusive classes to develop life skills among school students periodically, for all the classes, on all the life skills, at varying levels of issues and concerns that the children may easily be involved. At the third level, as a natural consequence of understanding and working towards development of life skills in life skill classes, with all needed conviction, teachers could try to integrate 
them in different school subjects as they feel fit. This way, life skills education could be meaningfully achieved in all schools, for all children.

(d) Impact Assessment issues: An equally important concern in Life Skills Education Programme is the assessment of the impact of the life skills education programme. It is desirable and necessary that at the end of different periods of life skills education programme, they also need to be assessed. Ideally, students could be assessed at the basal level before mounting the life skills education programme. At different stages of the life skills education programme they could be assessed for the rate of change among them. It implies that there must be a set of assessment tools for each of the skills. Since, the entire life skills education programme covers all students at all levels, the tools must be amenable for administration at all levels. In fact, it is ideal that the training package could comprise the assessment tool also.

\section{Proposed Components and Processes of Life Skills}

The present author has used the following components of life skills in his training package (Murthy, C.G.V. and Rao, A.V.G. 2005). Different components enumerated are also operationally defined in the context of a skill. There are occasions certain components are present in more than one skill also. What is to be appreciated is that all the components acquire different relevance under the context of different skill.

\section{DECISION MAKING}

\section{Operational definitions of Components:}

1. Emotional Stability: is the ability to manage emotions and remain calm in any decisionmaking situation.

2. Objectivity: is the ability to describe decision-making situations/problems exactly as they are without being influenced by one's own or others' feelings, opinions and beliefs.

3. Self-knowledge: is the ability to make a realistic analysis of what one is capable of doing and what one is not capable of doing before making a decision.

4. Knowledge of the situation: is the ability to describe the context of the decision-making situation as well as its elements and their inter-relationships.

5. Analytical Ability: is the ability to identify elements of the given decision-making situation by analyzing it.

6. Divergent Thinking Ability: is the ability to think of diverse courses of action in any decision-making situation.

7. Synthetic Ability: is the ability to synthesize different available possible courses of action and to generate one's own course of action in decision-making situations.

8. Anticipation of consequences: is the ability to predict the possible consequences of choosing a possible course of action. 
9. Logical Thinking: is the ability to think systematically and sequentially using valid principles of logic.

10. Rationality: is the ability to take decisions or arrive at a conclusion on the basis of valid reasons or logic and NOT impulsively or by trial and error or on the basis of one's own feelings, opinions and beliefs.

11. General Intelligence: is the general mental ability to understand, think, learn and apply relevant procedures in decision-making situations.

Process of Decision Making: There are different models which propose different decision making processes. Only four models are listed below. Any model can be used for developing decision making skills through different interventions of different components.

Example 1 (Debord,K. and Canu,R.F. $\quad$ Example 2 (Robert, H. 1998) 1997)

1. Identify the decision to be made together with the goals it should achieve.

2. Gather information about the problem and your options.

3. List your options.

4. Write down the things that are not important to you in making your decision.

5. List 'pros \& cons about each option.

6. Compare your list of 'pros' \& 'cons' for each option with the things most important to you. Decide on the best overall solution.

7. Make your decision.

Example 3 (Mahalingam. 2004)

1. Define the problem.

2. Re-evaluate the situation.

3. Gather information.

4. Think of alternatives.

5. Choose an alternative.

6. Put your decision to work
Example 4 (Arshram,H. 1996)

1. Identification of the problem.

2. What is the goal?

3. Possible actions.

4. Predict outcome.

5. Pick the best alternative.

6. Implement the decision

\section{PROBLEM-SOLVING}

\section{Operational definitions of Components:}

1. Self knowledge: is the ability to understand and learn about one's own capacities, emotionalities, and actions in dealing with problems in various_situations of life.

2. Positive attitude: is the ability of being hopeful and confident of approaching the problems in life. 
3. Divergent thinking: is the ability to think in multiple ways, views and manage a problem from different perspectives.

4. Objectivity: is the ability to view one's own problem from a third person's perspective as they are.

5. Rationality: is the ability to solve problems based on reasons and logic than using crude methods or trial and error.

6. Logical thinking: is the ability to understand and deal with the problem in a systematic and orderly fashion.

7. Analytical ability: is the ability to break down and understand various aspects / components of the problem.

8. Synthetic ability: is the ability to put together and understand various aspects / components of the problem.

9. Anticipation of the consequences: is the ability to infer/foresee the after-effects of the problem and / or the possibilities of the success/failure of the strategies used in the management of the problem.

Process of Problem Solving: There are different models proposed by different people. The model proposed by Powell (1992) is as follows.

Powell has suggested 5 Stages of Problem Solving.

(a) Identifying and defining clearly what the problem is.

(b) Brainstorm.

(c) Decide which options are realistic and unrealistic from the list made in stage 2.

(d) Choose the option that is both most rewarding and feasible.

(e) Prepare and plan strategies for the accomplishment of chosen option.

\section{CREATIVE THINKING}

\section{Operational definitions of Components:}

1. Divergent Thinking: is the ability to think in different ways on one issue.

2. Innovativeness: is the ability to think in new ways of doing things, different from the routine ways, which is cost-effective if implemented, and worthy of emulation.

3. Novelty: is the ability to generate new ways of thinking which the individual / group had not used in the past.

4. Fluency: is the ability to generate fluently many ideas about a situation without any discontinuity of thoughts.

5. Flexibility: is the ability to shift perspectives while thinking and generate as many as ideas as possible.

6. Originality: is the ability to think very differently from the large majority of people on different social situations, disregard of their social approval.

7. Elaboration: is the ability to think in such a way where one is capable of expanding different issues to different proportions in a connected manner. 
8. Unconventionality: is the ability to think very differently from the practiced conventional ways of thinking by the group on any issue of social life.

9. Independence: is the ability to think without being influenced by the views of others.

Process of Creative Thinking: Wallas (1926) described a four-stage theory of creativity. They are as follows.
a. Preparation
b. Incubation
c. Illumination
d. Verification

\section{CRITICAL THINKING}

\section{Operational definitions of Components:}

1. Analytic and synthetic abilities: are the abilities to analyse different components of a social and personal situation and put them together meaningfully in order to understand the situation better by weighing different pros and cons.

2. Objectivity: is the ability to analyze social and personal situations based on pros and cons dispassionately by delinking personal feelings and subjectivity.

3. Anticipation of consequences: is the ability to anticipate the consequences of any line of thinking in social and personal situations.

4. Intelligence: is the general ability to weigh the strengths and weaknesses of any situation in the process of its understanding.

5. Logical Thinking: is the ability to think and reason systematically on all social and personal situations of life based on objective principles. The two methods of reasoning include inductive and deductive modes.

\section{Process of Critical Thinking:}
a. Perception of the situation
b. Analysis of the situation (logical)
c. Evaluation of situation for merits and demerits/ Pros and cons
d. Anticipate consequences
e. Reaching a conclusion (Murthy, C.G.V.2005)

\section{EFFECTIVE COMMUNICATION}

\section{Operational definitions of Components:}

1. Analytic ability - is the ability to analyse different components of a piece of information, in order to understand its content.

2. Synthetic ability - is the ability to integrate different pieces of information available in different domains, thereby creating a meaningful picture of different sets of information into an organized whole.

(C) The International Journal of Indian Psychology, ISSN 2348-5396 (e)| ISSN: 2349-3429 (p) | 68 
3. Expressive Skills - is the ability to present one's thoughts / ideas / feelings as effectively as possible through the use of spoken or written language apart from/along with the use of gestures.

4. Non-verbal Skills - is the ability to express and understand thoughts/ideas/feelings through body postures, facial expressions and actions without the use of language.

5. Postures - is the general way of holding the body, especially back, shoulders and head when standing, walking or sitting which keep conveying some meaning.

6. Gestures - is the ability to use the movement of the body appropriately, especially hands and arms to enhance the intent of communication.

7. Presentation - is the ability to express thoughts /ideas /feelings formally as suitable to the demands of different types of situations.

8. Assertiveness - is the ability to put across one's views persuasively with a strong sense of conviction.

9. Creativity - is the ability to use different alternative ways to reach out to people suitably and successfully.

10. Objectivity - is the ability to participate in a communicative situation without any preconceived notions about persons involved or the content of communication.

11. Sensitivity - is the ability to be sensitive to the feelings of others in social situations in order to communicate effectively.

12. Patient Listening - is the ability to receive auditory inputs with full respect in a sustained manner in any conversation, without interrupting others until they are completed.

13. Imaginability - is the ability to foresee consequences of a communicative situation, which enables one to modulate the communication suitably.

14. Reacting on the spur of the moment: is the ability to react to any situation instantaneously, making sense.

\section{Process of Effective Communication:}

a. Identifying the need for communication:.

b. Thinking of suitable medium for communicating: language, gestures, audio-visual support etc.

c. Encoding the messages in the medium identified:

d. Planning support system:

e. Presentation:

f. Observing and looking for feedback:

g. Modifying the encoded message so as to overcome the gaps observed in the first attempt at communicating:

h. Modifying the encoded message so as to overcome the gaps observed in the first attempt at communicating: (Kumaraswamy, H. 2005) 


\section{INTERPERSONAL RELATIONSHIP}

\section{Operational definitions of Components:}

1. Empathy: is the ability to feel with others in social situations which can lead to the development of good interpersonal relationships.

2. Sympathy: is the ability to feel for others in social situations which can lead to the development of good interpersonal relationships.

3. Sensitivity: is the ability to be sensitive to the feelings, emotions and needs of others in social situations which can lead to the development of good interpersonal relationships.

4. Tolerance: is the ability to endure and respect feelings, views, attitudes etc, of others in interpersonal interactions irrespective of our agreement or disagreement on them.

5. Positive Attitude: is the ability to see the positive aspects in others and appreciate them in interpersonal relationships.

6. Accepting others as they are: is the ability to accept others with their strengths and weaknesses as it exists without showing any personal bias or prejudice about them in interpersonal relationships.

7. Reciprocality: is the ability to demonstrate a healthy attitude of give and take in social situations with others while interacting.

8. Etiquette: is the ability to show behaviour that is appropriate to different social situations which can earn respect and facilitate good interpersonal relationships.

9. Healthy Distance: is the ability to take only that much of liberty as the relationship empowers and demands and not misuse the liberty between any two individuals in any social situation.

10. Lack of Prejudices and Stereotypes: is the ability to interact with people without being governed by preconceived notions about individuals /groups in any social situation.

11. Effective Communication: is the ability to express the thoughts and feelings in nonthreatening ways.

\section{Process of Interpersonal Relationships:}

a. Willingness based on some assessment

b. Positive Attitude

c. Effective Communication

d. Etiquette

e. Sensitivity

f. Accepting others as they are

g. Reciprocality

h. Healthy Distance (Dhanya, P.V.P. and Rao, A.V.G. 2005) 


\section{SELF AWARENESS}

\section{Operational definitions of Components:}

1. Identifying strengths and weaknesses: is the ability to identify one's own strengths as well as weaknesses without any personal bias or prejudices.

2. Objectivity: is the ability to understand one's own strengths, weaknesses, emotions and feelings against a set of criteria.

3. Introspectionability: is the ability to assess one's own behaviour for their appropriateness or inappropriateness /adequacy or inadequacy shown in different life situations.

4. Accepting self as it is: is the ability to accept one's own self as a whole, in terms of his/her strengths and weaknesses, which makes the person unique.

5. Openness: is the ability to welcome thoughts or opinions from others if they are useful, despite they being contradictory to one's own initial conviction or belief.

6. Reflectivity: is the ability to use processes of seeing, understanding, pausing and evaluating one's own self in relation to his strengths and weaknesses in order to improve one self.

\section{Process of Self Awareness:}
a. Develop objectivity in life:
b. Avoid being narcissistic:
c. Learn to be realistic in life:
d. Assess your own strengths and weaknesses:
e. Shun developing complexes about yourself:
f. Verify your traits based on different evidences:
g. Accept yourself with all positive and negative attributes: (Murthy, C.G.V. 2005)

\section{EMPATHY}

\section{Operational definitions of Components:}

1. Sensitivity: is the ability to sense the feelings, needs, emotions and actions of other people in social situations.

2. Objectivity: is the ability to assess the requirement of assistance to others in need, in society, excluding one's own personal biases and prejudices towards the individual or the group.

3. Social Inclination: is the ability to develop and show the attitude that as a member of the society one has to do something for the welfare of the society and its members as and when situation arises.

4. Social Responsibility: is the ability to feel responsible for the society by way of understanding the feelings, needs, emotions and actions of people in a social situation and also contribute to the welfare of society and its members.

5. Social Obligation: is the ability to feel that it is one's duty to understand the feelings, needs, actions and emotions of people in society where one lives and extend help voluntarily in different situations without even being asked for. 


\section{Process of Empathy:}

a. Perception of the situation

b. Analysis of the situation

c. Action / Decision about the involvement (Herle, N; Nagaraja, C.G. and Murthy, C.G.V.2005)

\section{COPING WITH EMOTIONS}

\section{Operational definitions of Components:}

1. Recognising: is the ability to identify or become aware of one's own feelings, the course or origins of those feelings.

2. Empathy: is the ability to feel with others in different emotional situations.

3. Sympathy: is the ability to feel for others in different emotional situations.

4. Objectivity: is the ability to understand the emotions as they are and not based on personal biases.

5. Emotional Intelligence: is the ability to identify one's own feelings, feelings of others, regulate one's emotions and handle situations involving different emotions.

6. Self-Awareness: is the ability to be aware of internal changes and different reactions given to different emotional situations in social and personal situations.

7. Analytic and Synthetic ability: Analytic ability refers to evaluating the situation in terms of what has led to the emotional situation, and what would be the likely result. Synthetic ability refers to the ability to organise and use the appropriate emotions in response to the emotional situations.

8. Sense of Proportion: is the ability of an individual to be aware of the intensity of his emotional reaction and be able to sense whether the same is required for the situation.

9. Emotional Shock Absorbers: is the ability to withhold any emotional shock potential of disturbing the individual directly, by trying to be non emotional, rational and capable of understanding the sources and meaning of emotionally disturbing news.

10. Resilience: is the ability to recover quickly from any emotionally disturbing situation and get back to mental cheerfulness.

\section{Process of Coping with Emotions:}

a. Recognition that an emotion has impacted on the individual.

b. Analysing the situation objectively

c. Retaining a sense of proportion

d. Analysing one's abilities and inabilities

e. Using emotional shock absorbers

f. Resilience (Venkatesan, S. 2005) 


\section{COPING WITH STRESS}

\section{Operational definitions of Components:}

1. Recognisability: is a group of abilities to identify or become aware of one's own feelings of stress, the course or origins of that experience of stress, an appraisal of the barriers or obstacles that seemingly impede resolution of the stress as well as setting of goals appropriate and adequate for bringing about a relative end to ongoing stressful experiences.

2. Planning ability: is a group of abilities to draw blueprints or schemes for enabling short term or long term future courses of action by including effective consideration towards the act, manner or practice of handling time constraints within the gamut of planning for coping or bringing about a relative end to ongoing stressful experiences.

3. Objectivity: is an ability to be in a state, quality or an attitude of being impersonal, external or uninvolved to a problem or situation being examined or under focus.

4. Empathy: is an ability to develop identification with or understanding of another person's situation, feelings and motives.

5. Resilience: is the ability to maintain mental cheerfulness by recovering quickly from change or misfortune.

6. Reality Orientation: is the ability to perceive or be aware of the objective world in relation to one's self across place, time or person.

7. Self-awareness: is the ability to develop realization of oneself as an individual entity or personality, including one's feelings, traits or behaviours covering both good and bad.

8. Relaxationability: is the ability to rejuvenate or refresh one's body and mind so as to bring it back to an optimal state of functioning.

9. Entertainability: is the ability to amuse, please or indulge in diversions that enables the individual to get back to optimal state of functioning.

10. Stability: is the ability of being constant, firm, steadfast and resistant to change.

\section{Process of Coping with Stress:}

a. Recognition that stressor has impacted the individual

b. Recognizing the source of stress

c. Understand stress

d. Assessing the extent of the intensity of stress

e. Reality orientation

f. Identify what can make the individual relax

g. Try relaxation activities and learn to handle stress (Venkatesan, S. 2005)

The above operationally defined components can provide clarity, which need to be understood as basic bricks of skill development activity. For each of the components, a number of interventions need to be developed. The choice of the interventions/ techniques must be such, which are 
Issues, Problems and Possibilities of Life Skills Education for School Going Adolescents

doable by all teachers. Keeping in view this and the suggestions given by the WHO, different interventions are to be selected.

\section{Techniques of developing Life Skills?}

Development of life skills can be effectively done using different techniques. WHO recommends brainstorming and Role-play. Both need to be followed by discussion.

(A) Brainstorming: is a technique widely used in different contexts and one such is in the development of life skills. It is a technique, where, all the members of a group will have a common topic on which, they can discuss freely. There is an initiator for this. In a school context, it could be a teacher. While different ideas are being given out by participants, no value judgment is made, no comments are passed, no agreements or disagreements are suggested, and no appreciation is shown to any of the issues. The task of the facilitator is only to pool as many ideas as given by members. After, the group feels that it has exhausted the entire set of issues expressed, it could then be put to discussion. For example, there is a problem for which a group might feel like subjecting it to brainstorming to pool all possible solutions. In this process;

(a) Identify all those issues, which are not realistic and possible in practical situation.

(b) Rearrange ideas so that it gives some order, sequence or trend about an issue.

(c) Arrive at the consensus of all the members on the rearranged set of issues.

(B) Role Play: Role play is yet another technique used in different contexts and one such is in the development of life skills. Role play is a technique where a group of people or students in a school context are encouraged to enact a small skit based on a theme or script. In this process children develop certain views about the worth of the context. When the role-play is enacted, the entire class watches the play. A specific situation is depicted. After this enactment, involve all the students in discussion. Discuss the content and central feature of the role-play, focus discussions on the skill and the specific component of the life skill. This enables students to understand the importance of the skill. It makes them think about the concern of the skill and enables them to work towards it. Therefore, it not only sensitizes one to skill but also provides opportunity to develop when students are exposed to many such role-play situations.

Epilogue: Life Skills Education has indeed attracted the imagination of many concerned professionals and institutions. Perhaps since the WHO's initiatives, India could have taken a definite stand on the role of life skills education in school education. Consequently, there are different views and interpretations of life skills education in the country. Perhaps it is not too late to take a call on building consensus towards bringing a certain kind of common understanding of how life skills needs to be promoted in the country. 


\section{REFERENCES}

Arshram,H. (1996). Leadership decision making: Ethics and decision making. http: //home.ubalt.edu/ntsbarsh/opre640/partXIII.htm\#rethics

Botvin, G.J., Baker, E., Botvin, E.M.. Filazzola, A.D. and Millman, R.B. (1984). Alcohol abuse prevention through the development of personal and social competence: A pilot study. Journal of Studies on Alcohol, 45, 550-552.

Botvin, G.J., Eng, A. and Wlliams, C.L. (1980). Preventing the onset of cigarette smoking through Life Skills Training. Preventive Medicine, 11, 199-211.

Debord, K. and Canu, R.F. (1997). Decision making: It's a skill. http://www.ces.ncsu.edu/depts/fcs/human/pubs/wfdecion.html

Dhanya, P.V.P. and Rao, A.V.G. (2005). Interpersonal Relationships. In Murthy, C.G.V. and Rao, A.V.G. (2005). (Eds) Life Skills Education: Training Package. Mysore: Regional Institute of Education.

Gonzalez, R. (1990) Ministering intelligence: A Venezuelan experience in the promotion of cognitive abilities. International Journal of Mental Health, 18 (3). 5-19.

Herle, N; Nagaraja, C.G. and Murthy, C.G.V. (2005). Empathy. In Murthy, C.G.V. and Rao, A.V.G. (2005). (Eds) Life Skills Education: Training Package. Mysore: Regional Institute of Education.

Kumaraswamy, H. (2005). Effective Communication. In Murthy, C.G.V. and Rao, A.V.G. (2005). (Eds) Life Skills Education: Training Package. Mysore: Regional Institute of Education.

Mahalingam. (2004). How to develop your decision making skills. In, Good decision makers are successful people. http://www.hooah4health.com /spirit/decisions.htm

Murthy, C.G.V. (2005). Critical Thinking. In Murthy, C.G.V. and Rao, A.V.G. (2005). (Eds) Life Skills Education: Training Package. Mysore: Regional Institute of Education.

Murthy, C.G.V. (2005). Self awareness. In Murthy, C.G.V. and Rao, A.V.G. (2005). (Eds) Life Skills Education: Training Package. Mysore: Regional Institute of Education.

Murthy, C.G.V. and Rao, A.V.G. (2005). Life skills Education: Training Package (Parts A, B, $C)$. Mysore: Regional Institute of Education.

Olweus, D. (1990). A national campaign in Norway to reduce the prevalence of bullying behaviour. Paper presented to the Society for Research on Adolescence Biennial Meeting, Atlanta, December 10-12.

Pentz, M.A. (1983). Prevention of adolescent substance abuse through social skills development. In Glynn, TJ. et al. (Eds.) Preventing adolescent drug abuse: Intervention strategies., NIDA Research Monograph No 47 Washington DCNIDA, 195-235.

Powell, T. (1992). The Mental Health Handbook. Oxon: Winslow Press

Prutzman, P., Stern, L. Burger, M.L., and Bodenhamer, G. (1988) The friendly classroom for a small planet: Children's creative response to conflict program. New Society Publishers, Santa Cruz, USA.

Roberts, R. (1998). 7 steps decision making model. http://www.unf.edu/dept/cdc/ 
Schinke S P (1984) Preventing teenage pregnancy. In Hersen, M., Eisler, R.M. and Miller, P.M. (Eds.) Progress in behaviour modification. New York: Academic Press, 31-64. service/decision.htm

TACADE (1990). Skills for the primary school child: Promoting the protection of children. Salford, UK.

Venkatesan, S. (2005) Coping with emotions. In Murthy, C.G.V. and Rao, A.V.G. (2005). (Eds) Life Skills Education: Training Package. Mysore: Regional Institute of Education.

Venkatesan, S. (2005) Coping with stress. In Murthy, C.G.V. and Rao, A.V.G. (2005). (Eds) Life Skills Education: Training Package. Mysore: Regional Institute of Education.

Wallas (1926). http://members.optusnet.com.au/charles57/Creative/Brain /wallis. htm WHO. (1997). Life Skills Education in schools. Geneva: Division of Mental Health.

WHO/GPA. (1994) School Health Education to Prevent AIDS and STD: A Resource Package for Curriculum Planners. World Health Organization / Global Programme on AIDS, Geneva.

Zabin, L.S. Hirsch, M.B., Smith, E.A., Streett. R. and Hardy. J.B. (1986). Evaluation of a pregnancy prevention programme for urban teenagers. Family Planning Perspectives, 18. 119-126.

How to cite this article: V Murthy (2016), Issues, Problems and Possibilities of Life Skills Education for School Going Adolescents, International Journal of Indian Psychology, Volume 3, Issue 3, No. 11, DIP: 18.01.196/20160303, ISBN: 978-1-365-21307-6 